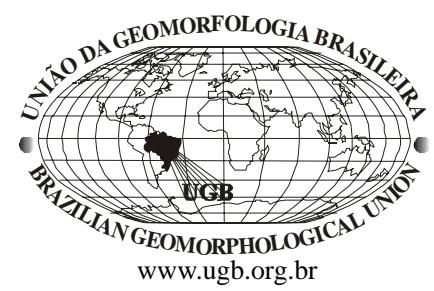

NOTA TÉCNICA

\title{
GEOMORFOLOGIA BRASILEIRA: PANORAMA GERAL DA PRODUÇÃO NACIONAL NO INÍCIO DO SÉCULO XXI (2001-2005)
}

\author{
André Augusto Rodrigues Salgado
}

Professor Adjunto do Departamento de Geografia da UFMG geosalgado@yahoo.com.br Departamento de Geografia - Instituto de Geociências - Universidade Federal de Minas Gerais - Av. Antônio Carlos, 6.627 Pampulha 31270-901 Belo Horizonte - MG. Telefone: (31) 3499-5438.

Joseane Biazini

Graduanda em Geografia na UFMG jbiazini@yahoo.com.br

Sebastian Hennig

Pesquisador do Departamento de Geografia da Leipzig Universität - Alemanha sebastian_hennig@hotmail.com

Faculdade de Geografia - Johannisallee 19 a - Phone: +49 (0) 341/97-38609

\begin{abstract}
Resumo
O presente trabalho, através da análise dos artigos publicados em periódicos nacionais e internacionais, traça um panorama da geomorfologia brasileira nos cinco primeiros anos do século XXI. Os resultados obtidos indicam a existência de centros de excelência nacional em algumas das especialidades da geomorfologia. Indicam ainda que a produção brasileira concentra-se no centro-sul do país e que o ramo mais desenvolvido é o da geomorfologia fluvial. Por fim, apontam para o fato de que o amadurecimento do trabalho dos centros nacionais de excelência nos diversos ramos da geomorfologia pode fazer com que a produção geomorfológica brasileira aumente de importância no cenário internacional.
\end{abstract}

Palavras-chave: Geomorfologia, Produção de artigos científicos, Brasil.

\begin{abstract}
This work, based on analysis of the scientific papers published in national and international journals, verifies the panorama of brazilian geomorphology in the five first years of $21^{\circ}$ century. The results shows that, in some subjects, there are national's research centres with important production and show also that the brazilian production is concentrated in the centre-south of the country and that the fluvial geomorphology is the subject more developed. Finally, show that the sequence of the work, in the many subjects, of the research centres more developed can increase the importance of brazilian geomorphology in the international scientific scenario.
\end{abstract}

Keywords: Geomorphology, Production of scientific papers, Brazil. 


\section{Introdução}

Os periódicos científicos, devido ao fato de possuírem amplo alcance e serem os principais veículos de divulgação de trabalhos científicos, constituem-se, atualmente, como as forças motrizes da ciência e, conseqüentemente, determinam os paradigmas que orientam sua evolução. Esta constatação é válida também para a geomorfologia (Doyle \& Julian, 2005). Logo, a análise dos artigos publicados em periódicos especializados em geomorfologia constitui uma ferramenta notável para se traçar, com relativa segurança, um panorama geral da produção científica de caráter geomorfológico (Dorn, 2002).

Neste contexto, insere-se o presente trabalho que visa, através da análise dos artigos publicados em periódicos (nacionais e internacionais) por profissionais radicados em centros de pesquisa e universidades brasileiras, um panorama geral da geomorfologia brasileira.

\section{Procedimentos da Pesquisa}

O método adotado neste trabalho baseia-se em revisão bibliográfica. Esta revisão é realizada em dois tipos de periódicos especializados em geomorfologia: (i) nacional e; (ii) internacional.

A Revista Brasileira de Geomorfologia, editada pela União Brasileira de Geomorfologia, única revista nacional especializada em geomorfologia, classificada em 2006 pela CAPES (Geografia Física) como de abrangência de circulação nacional, nível de qualidade A (mais elevada), é utilizada para se traçar um panorama da produção brasileira no âmbito nacional. Os periódicos Geomorphology (editado pela Associação Internacional de Geomorfologia), Earth Surface Processes and Landforms (editado pela União Britânica de Geomorfologia) e Zeitschrift für Geomorphologie (editado pela Associação Alemã de Geomorfólogos), foram selecionados para se traçar o panorama da produção brasileira no âmbito internacional. Estes três periódicos foram selecionados em razão de que, além de serem classificados como internacionais A, segundo a CAPES (Geografia Física em 2006), constituem os únicos especializados em geomorfologia que são acompanhados pelo Journal Citation Reports (Journal Citation Reports 2001, 2002, 2003, 2004, 2005), jornal este que determina o fator de impacto de uma revista científica.

Para a produção publicada no Brasil os parâmetros analisados foram: (i) centro de pesquisa e/ou universidade de filiação dos autores e co-autores; (ii) tema do artigo e; (iii) cooperação nacional e internacional. Para os artigos internacionais, além dos mesmos parâmetros, foi avaliada percentualmente a produção bibliográfica nacional em relação a mundial.

A filiação dos autores e co-autores foi determinada pelo endereço institucional e ou de correspondência indicado no artigo. Não foram consideradas a nacionalidade do autor/co-autor, e sim o fato do mesmo trabalhar em instituição brasileira. Logo, pesquisadores estrangeiros que tenham publicado enquanto ligados a centros de pesquisa e universidades nacionais foram computados. Ao contrário, pesquisadores brasileiros que tenham publicado por instituições internacionais não foram computados. Quanto a artigos com co-autores de instituições diferentes, os mesmos foram computados para as duas instituições. Conseqüentemente, o número de autores e instituições, supera o de artigos.

Para se classificar os artigos por especialidades da geomorfologia, primeiramente foram levantados os objetivos principais de cada artigo. De acordo com estes objetivos os artigos foram classificados em uma das sub-divisões a seguir: (i) Evolução regional do relevo de áreas áridas e semi-áridas; (ii) Evolução regional do relevo de áreas tropicais úmidas e semi-úmidas; (iii) Evolução regional do relevo de áreas subtropicais; (iv) Evolução regional do relevo de áreas glaciais e peri-glaciais; (v) Geomorfologia fluvial; (vi) Geomorfologia costeira e submarina; (vii) Geomorfologia cárstica; (viii) Discussões teóricas, incluindo epistemológicas e ensaios de metodologias e; (ix) Outros, que engloba análises ambientais, mapeamentos, aplicação de modelos, geoprocessamento aplicado a geomorfologia, processos erosivos acelerados em vertentes e etc. Deve-se ressaltar que esta classificação dos artigos em especialidades da geomorfologia por vezes se baseou em critérios subjetivos, visto que muitos trabalhos situavam-se na fronteira entre duas temáticas diferentes.

O parâmetro cooperação visou avaliar se o trabalho publicado foi resultado de cooperação entre pesquisadores de diferentes instituições. Logo, neste parâmetro, os artigos podem ser sub-divididos em: (i) resultados de esforços de pesquisador(es) de uma única instituição; (ii) resultado de cooperação entre duas ou mais instituições nacionais e; (iii) resultado de cooperação entre pesquisadores/equipes nacionais e internacionais.

Convém ressaltar que, neste trabalho, não avaliamos o mérito científico dos artigos publicados nos periódicos analisados. Esta avaliação foi realizada pelos consultores e editores desses periódicos. Foram eles que consideraram esses artigos com mérito suficiente para a publicação em seu periódico. Igualmente, não coube, neste momento, uma análise teórico-metodológica ou epistemológica dos artigos publicados. 
Por fim, no que concerne aos procedimentos desta pesquisa, deve ser ressaltado que este trabalho não objetiva compreender o enquadramento teórico dos artigos e periódicos investigados. Tão pouco objetiva enquadrar a relação destes artigos em termos de escolas de pensamento e da evolução histórica das geociências. Os procedimentos utilizados nesta pesquisa objetivaram somente traçar o recente panorama da produção geomorfológica brasileira nos periódicos nacionais e internacionais de maior impacto na comunidade geomorfológica.

\section{Resultados}

\section{Produção de caráter nacional}

A Revista Brasileira de Geomorfologia publicou nos cinco primeiros anos do século XXI, 43 artigos que tiveram autore(s) e/ou co-autore(s) ligados a instituições brasileiras. A análise da Figura 1 demonstra que esta produção se concentrou nas temáticas: (i) Geomorfologia fluvial; (ii) Outros; (iii) Geomorfologia costeira e submarina; (iv) Evolução regional do relevo de áreas tropicais úmidas e semi-úmidas e; (v) Discussões teóricas.

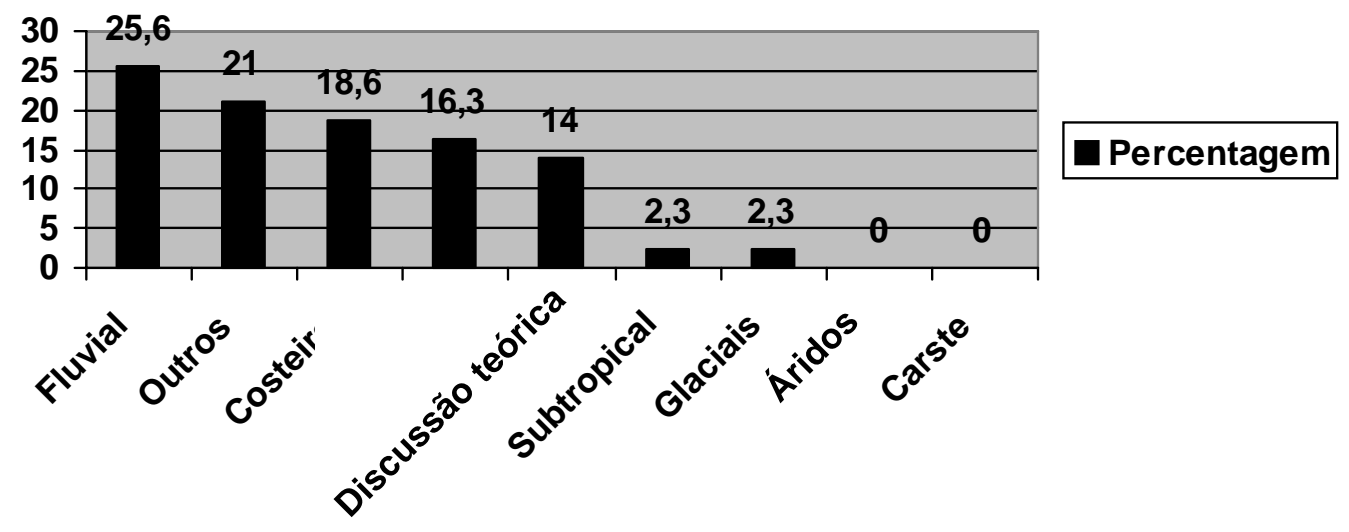

Figura 1 - Produção de artigos nacionais por temas.

Os autores dos artigos publicados são em sua quase totalidade oriundos de instituições das regiões sudeste, centro-oeste e sul, sendo que menos da metade dos estados da federação tiveram autore(s) e/ou co-autore(s) produtores de artigos (Figura 2). Já em termos de centros de pesquisa destacaram-se como grandes produtores a Universidade Federal do Rio de Janeiro, a UNESP - Rio Claro e a Universidade de Guarulhos, ambas do Estado de São Paulo e a Universidade Estadual de Maringá do Estado do Paraná (Figura 3).

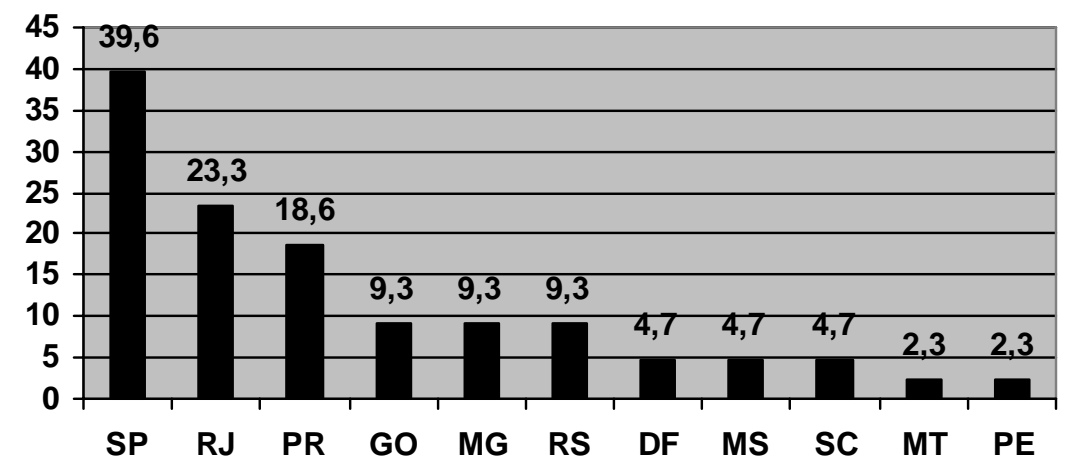

Percentagem

Figura 2 - Produção de artigos nacionais em geomorfologia por estado da federação. 


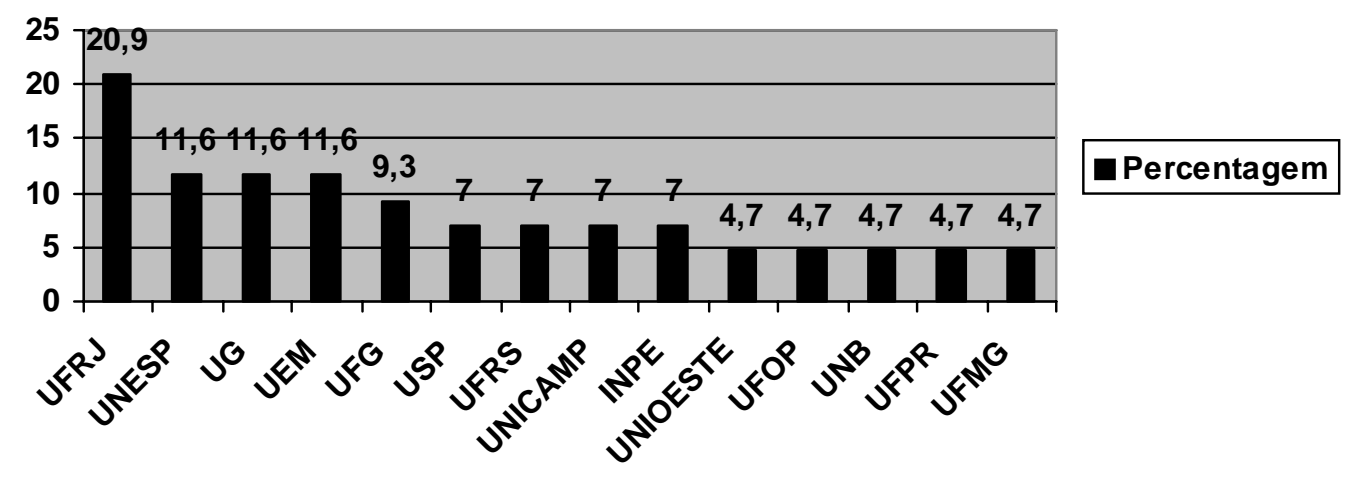

Figura 3 - Principais centros de pesquisa produtores de artigos nacionais de geomorfologia.

A análise por tema dos artigos oriundos dos seis estados que se constituíram como maiores produtores - São Paulo, Rio de Janeiro, Paraná, Minas Gerais, Goiás e Rio Grande do Sul - demonstra que São Paulo se destaca na geomorfologia fluvial vinculada principalmente a UNESP - Rio Claro e à Universidade de Guarulhos, e em discussões teóricas (Universidade de São Paulo). O Estado do Rio de Janeiro na geomorfologia costeira e em discussões teóricas (teste de novas metodologias), principalmente vinculados à Universidade Federal do Rio de Janeiro. $\mathrm{O}$ Estado do Paraná na geomorfologia fluvial, com destaque para a produção da Universidade Estadual de Maringá. O
Estado de Minas Gerais na evolução regional do relevo de áreas tropicais úmidas e semi-úmidas vinculados à Universidade Federal de Minas Gerais e à Universidade Federal de Ouro Preto, e em discussões teóricas vinculados à Pontifícia Universidade Católica de Minas Gerais. Por fim, o Estado de Goiás se destaca na geomorfologia fluvial (Universidade Federal de Goiás).

A Figura 4 mostra que a maior parte dos artigos publicados no Brasil não é fruto de cooperação entre diferentes instituições, sendo que, aqueles que são resultado de cooperação, em sua maioria, não possuem autores oriundos de instituições estrangeiras.

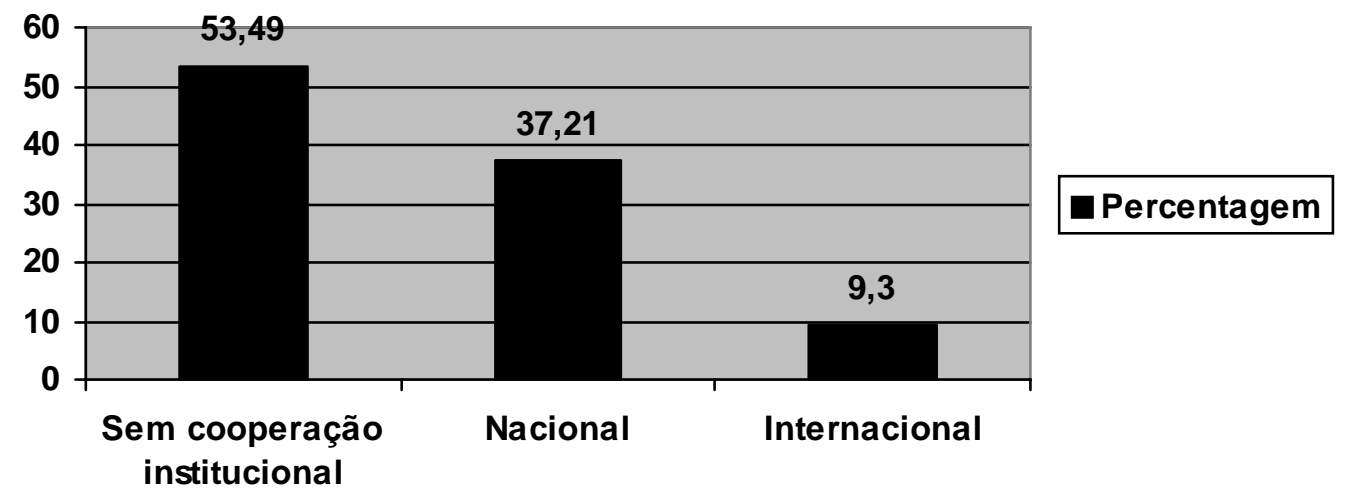

Figura 4 - Cooperação na produção de artigos nacionais.

\section{Produção de caráter internacional}

No âmbito internacional a produção brasileira ainda é diminuta (Tabela 1) e se concentra na geomorfologia fluvial, visto que dos 24 (vinte e quatro) artigos publicados, 12 (doze) tratam deste tema. Em segundo lugar, mas com importância bem menor do que a geomorfologia fluvial, está a geomorfologia costeira, seguida de perto pela evolução regional do relevo tropical. Na seqüência, mas com menos de $10 \%$ de representatividade, aparecem a geomorfologia cárstica, as temáticas agrupadas como outros e a evolução regional do relevo de áreas áridas e semiáridas (Figura 5). 
Tabela 1 - Produção brasileira de artigos internacionais em relação ao total mundial.

\begin{tabular}{|l|r|r|r|r|r|r|}
\hline & 2001 & \multicolumn{1}{|c|}{2002} & $\mathbf{2 0 0 3}$ & \multicolumn{1}{c|}{$\mathbf{2 0 0 4}$} & $\mathbf{2 0 0 5}$ & \multicolumn{1}{l|}{ Total } \\
\hline Mundial* & 241 & 298 & 299 & 296 & 389 & 1523 \\
\hline Brasil & 1 & 10 & 2 & 5 & 6 & 24 \\
\hline Percentagem Brasil/Mundo & 0,4 & 3,4 & 0,7 & 1,7 & 1,5 & 1,6 \\
\hline
\end{tabular}

* Considerando os artigos publicados pelos periódicos internacionais: (i) Geomorphology; (ii) Earth, Surface, Processes and Landforms; (iii) Z. Geomorphologie. Foram contabilizados também os números suplementares e as edições especiais.

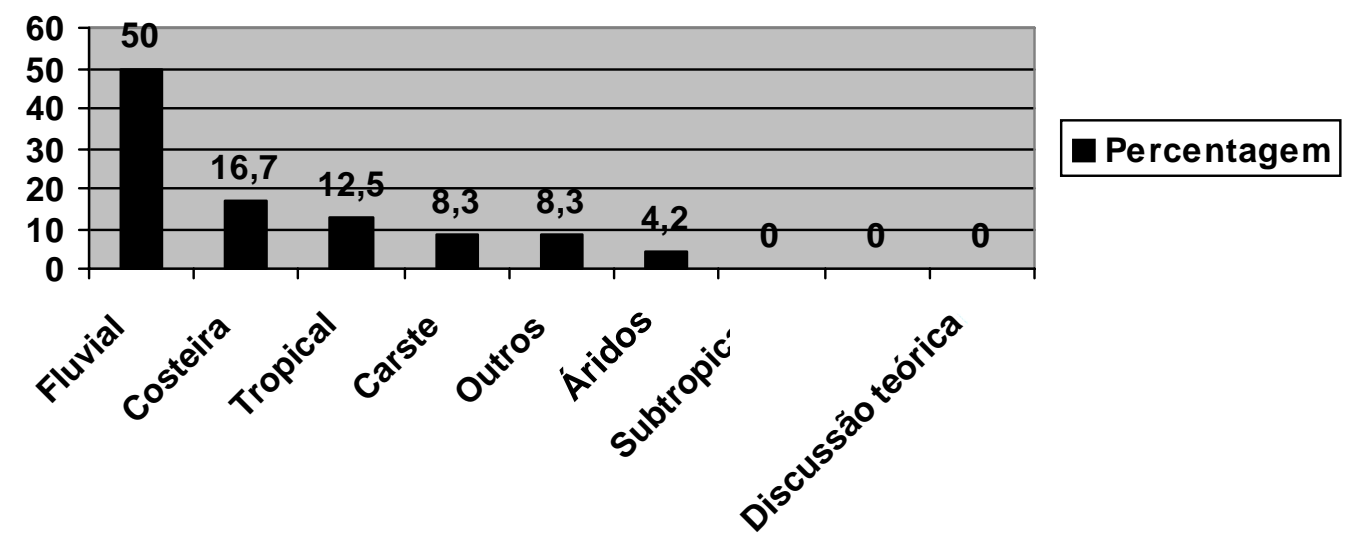

Figura 5 - Produção de artigos brasileiros em periódicos internacionais por temas.

Todas as regiões do Brasil produziram artigos internacionais de geomorfologia. Porém, a exemplo do que ocorreu na publicação de artigos nacionais, as regiões sul e centrooeste sudeste do país concentraram a maior parte da produção de artigos internacionais. Os estados que constituíram os maiores produtores foram, nesta seqüência, Goiás, Paraná e
Minas Gerais (Figura 6). Entretanto, esta concentração não foi tão evidente, visto que estados como o Amazonas e o Ceará apresentaram alta produção. Vale ressaltar que os dois maiores produtores de artigos nacionais - São Paulo e Rio de Janeiro - em artigos internacionais, apresentaram queda sensível na porcentagem de artigos publicados (Figura 6).

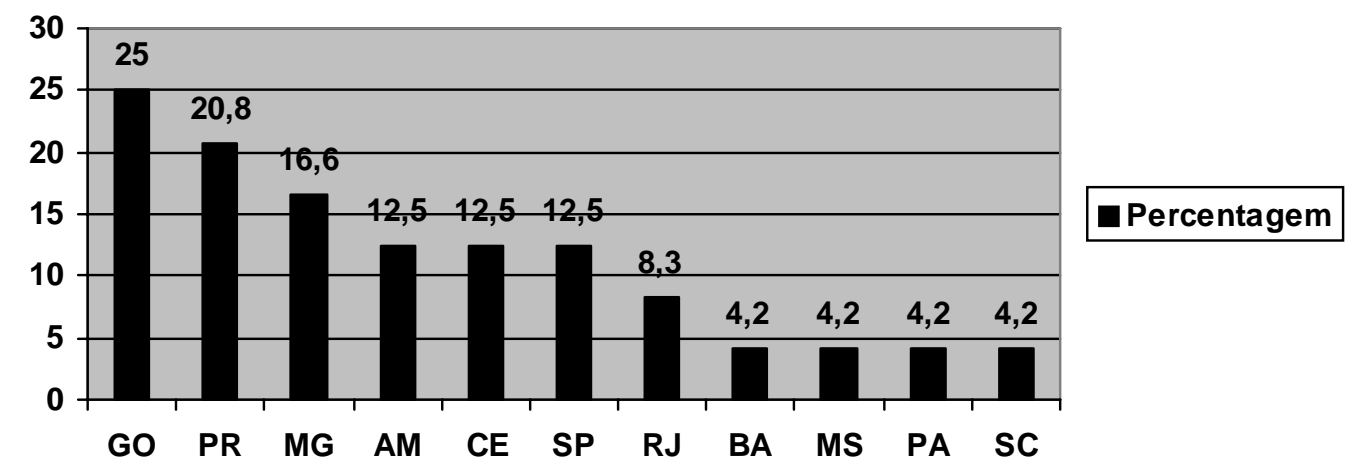

Figura 6 - Produção de artigos internacionais em geomorfologia por estado da federação.

Em termos de centros de pesquisa, como a produção brasileira em periódicos internacionais se concentrou na geomorfologia fluvial, os principais centros de pesquisa nacionais nesta temática (Figura 3) - Universi- dade Federal de Goiás, e Universidade Estadual de Maringá, bem como em menor escala, a Universidade de Guarulhos - acabaram por se constituir também como importantes produtores de artigos internacionais (Figura 
7). Apresenta ainda destaque na geomorfologia fluvial em publicações internacionais, a Universidade Federal do Amazonas. Fora do âmbito da geomorfologia fluvial, destacam-se a Universidade Federal do Ceará tratando temas diversos e a Universidade Federal de Minas Ge- rais com ênfase no Carste. Com mais de 5\% da produção nacional de artigos ainda merece ser citada a Universidade Federal do Rio de Janeiro, que em termos internacionais limitou suas publicações à evolução regional do relevo tropical.

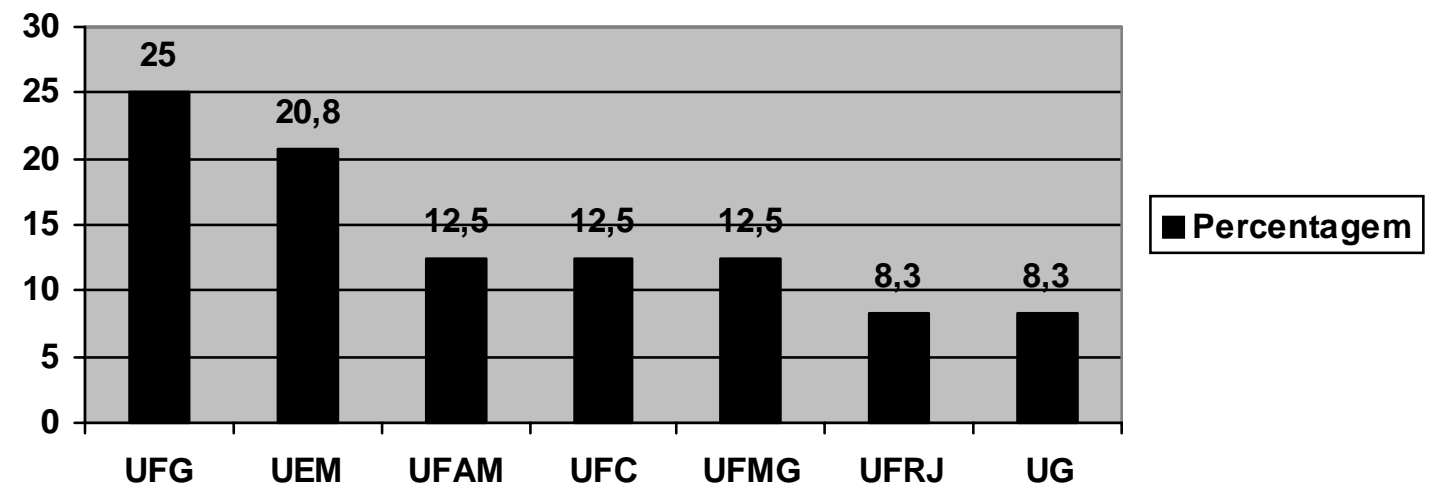

Figura 7 - Principais centros de pesquisa brasileiros produtores de artigos internacionais de geomorfologia.

Quanto ao item cooperação, é possível verificar que, ao contrário do que aconteceu com os artigos nacionais, a maior parte dos artigos internacionais é resultado de coope- ração entre profissionais de duas ou mais instituições diferentes (Figura 8). Principalmente entre equipes brasileiras e estrangeiras.

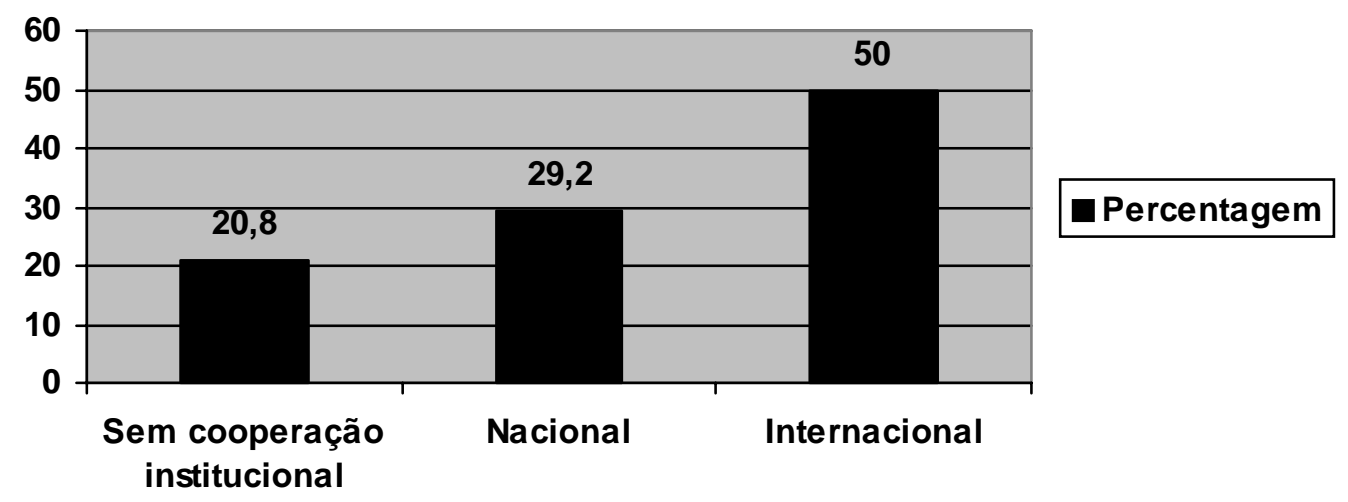

Figura 8 - Cooperação na produção de artigos nacionais.

\section{Discussão}

A Geomorfologia Fluvial constitui a especialidade que mais publicou na geomorfologia brasileira no período investigado (2001-2005). Pode-se mesmo afirmar que, em nível internacional, é o único entre eles em que a produção brasileira merece algum destaque, obtido principalmente por pesquisadores da Universidade Federal de Goiás e da Universidade Estadual de Maringá. Deste modo, embora seja possível afirmar que existem no Brasil outros centros de excelência internacional em geomorfologia fluvial, estes constituem situações de contribuições pontuais.
No que concerne à origem regional e estadual das instituições dos autores, merece destaque o fato de que, de maneira geral, nos periódicos nacionais e internacionais, os mesmos centros de pesquisa já citados foram identificados como os principais produtores e concentradores nas mesmas especialidades da geomorfologia. Este fato confirma que a Revista Brasileira de Geomorfologia é um bom periódico para se analisar a produção geomorfológica brasileira, visto que as tendências por ela mostradas são muito semelhantes àquelas evidenciadas nas revistas internacionais de maior impacto.

Ainda no que se refere à origem dos autores, principalmente no âmbito nacional, fica evidente uma preponderância 
dos estados mais ricos e populosos como, por exemplo, São Paulo, Rio de Janeiro, Minas Gerais e Paraná. Os estados localizados em outras regiões, sobretudo os do norte e nordeste, possuem a maior parte de suas publicações como frutos de cooperações com centros de pesquisas localizados nesses estados do centro-sul ou no exterior. Este foi o caso, respectivamente, do Amazonas e do Ceará que não apresentaram produção em periódico nacional, mas que graças a estas cooperações obtiveram uma boa produtividade de artigos internacionais. Evidentemente, tal realidade é conseqüência de fatores históricos e econômicos que dificultam o aparecimento de centros de excelência científica nas ditas áreas periféricas do país. Porém, o fato de boa parte dos artigos publicados em periódicos internacionais, ao contrário do que ocorreu com os nacionais, serem resultado de cooperação (Figuras 4 e 8), demonstra a necessidade por parte dos centros de pesquisa brasileiros de estabelecer contatos com outros centros, brasileiros e/ou estrangeiros, como forma de produzir trabalhos de alta qualidade. Tal necessidade não é válida somente para o norte e nordeste do Brasil. Na verdade, ela é mundial e ocorre em razão dos altos gastos para realização de pesquisa científica em geomorfologia, bem como da crescente necessidade de exames de ponta que confirmem as observações realizadas em campo.

\section{Conclusões}

A análise da produção geomorfológica brasileira através dos artigos publicados em periódicos especializados, apesar de fornecer dados incompletos, permite traçar um panorama geral recente desta ciência no Brasil, o que tornou possível traçar uma série de considerações finais para o período analisado (2001-2005):

1) A geomorfologia fluvial é a especialidade da geomorfologia que tem mais publicado no Brasil, tanto em termos de produção de artigos nacionais como internacionais;
2) Com exceção da geomorfologia fluvial, a produção brasileira de artigos científicos internacionais de geomorfologia é muito pequena;

3) As regiões centro-sul e sul do país concentram a maior parte da produção brasileira de artigos geomorfológicos;

4) É possível reconhecer centros brasileiros de excelência nacional nas diversas especialidades da geomorfologia, principalmente na fluvial;

5) Enquanto os artigos nacionais são, preferencialmente, frutos do trabalho de um único centro de pesquisa, os artigos internacionais são em sua maioria resultantes de cooperação científica internacional;

6) A cooperação entre diferentes centros de pesquisa constituiu o melhor caminho para que a geomorfologia brasileira fosse mais produtiva no período investigado (20012005).

\section{Referências Bibliográficas}

DORN, R. I. (2002) Analysis of geomorphology citations in the last quarter of 20th century. Earth Surface Processes Landforms, 27: 667-672.

DOYLE, M. N.; JULIAN, J. P. (2005) The most cited works in Geomorphology. Geomorphology, 72: 238-249.

JOURNAL CITATION REPORTS (2001) Journal Citation Reports Science Edition. London: Thomson.

JOURNAL CITATION REPORTS (2002) Journal Citation Reports Science Edition. London: Thomson.

JOURNAL CITATION REPORTS (2003) Journal Citation Reports Science Edition. London: Thomson.

JOURNAL CITATION REPORTS (2004) Journal Citation Reports Science Edition. London: Thomson.

JOURNAL CITATION REPORTS (2005) Journal Citation Reports Science Edition. London: Thomson. 